\title{
The Glutamate and Chloride Permeation Pathways Are Colocalized in Individual Neuronal Glutamate Transporter Subunits
}

\author{
Gregory P. Leary, ${ }^{1}$ Emily F. Stone, ${ }^{2}$ David C. Holley, ${ }^{1}$ and Michael P. Kavanaugh ${ }^{1}$ \\ Center for Structural and Functional Neuroscience, Departments of ${ }^{1}$ Biomedical and Pharmaceutical Sciences and ${ }^{2}$ Mathematics, University of Montana, \\ Missoula, Montana 59812
}

\begin{abstract}
Glutamate transporters have a homotrimeric subunit structure with a large central water-filled cavity that extends partially into the plane of the lipid bilayer (Yernool et al., 2004). In addition to uptake of glutamate, the transporters also mediate a chloride conductance that is increased in the presence of substrate. Whether the chloride channel is located in the central pore of the trimer or within the individual subunits has been controversial. We find that coexpression of wild-type neuronal glutamate transporter EAAT3 subunits with subunits mutated at R447, a residue governing substrate selectivity (Bendahan et al., 2000), results in transport activity consistent with two distinct noninteracting populations of transporters, in agreement with previous work suggesting that each subunit operates independently to transport substrate (Awes et al., 2004; Grewer et al., 2005; Koch and Larsson, 2005). In wild-type homotrimeric transporters, the glutamate concentration dependence of the anion conductance and the kinetics of glutamate flux were isolated and measured, and the anion channel activation was fitted to analytical expressions corresponding to (1) a central pore gated by binding to one or more subunits and (2) a channel pore in each subunit. The data indicate that glutamate-binding sites, transport pathways, and chloride channels reside in individual subunits in a trimer and function independently.
\end{abstract}

Key words: glutamate receptor; glutamate transport; chloride channel; modeling; uptake; EAAT

\section{Introduction}

In mammals including humans, high-affinity glutamate transporters are encoded by a group of five genes (EAAT1-5; SLC1A1$A 5)$ that are members of the dicarboxylate/amino acid:cation symporter family, which also includes the neutral amino acid transporters ASCT1 and ASCT2. This gene family is distinct from the family of genes encoding the transporters for neurotransmitters such as GABA, serotonin, dopamine, norepinephrine, glycine, and others. The excitatory amino acid transporters (EAATs) are found throughout the CNS and peripheral tissues; they are the major routes for cellular uptake of glutamate (Danbolt, 2001).

EAAT function is well described by a cyclical alternating access transport model in which L-glutamate (L-Glu) is cotransported with three $\mathrm{Na}^{+}$ions and one $\mathrm{H}^{+}$ion, followed by countertransport of one $\mathrm{K}^{+}$ion, restoring the initial state (Zerangue and Kavanaugh, 1996; Levy et al., 1998). In addition to mediating coupled glutamate transport, EAATs also exhibit a thermodynamically uncoupled chloride conductance that is increased in the presence of glutamate (Fairman et al., 1995; Picaud et al., 1995; Wadiche et al., 1995; Billups et al., 1996). The chloride conductance varies relative to the glutamate transport rate

Received Nov. 7, 2006; revised Feb. 7, 2007; accepted Feb. 8, 2007.

This work was supported by National Institutes of Health Grants NS33270 and P20 RR15583.

Correspondence should be addressed to Michael P. Kavanaugh, 301 Skaggs Building, University of Montana

Missoula, MT 59812. E-mail: michael.kavanaugh@umontana.edu.

D01:10.1523/JNEUROSCI.4851-06.2007

Copyright $\odot 2007$ Society for Neuroscience $\quad$ 0270-6474/07/272938-05\$15.00/0 among different EAAT subtypes $($ EAAT5 $\approx$ EAAT4 $>$ EAAT1 $>$ EAAT3 $>$ EAAT2). Whether the channel activity plays a physiological role in each of them is presently unclear, but the chloride conductance of the glutamate transporters on presynaptic terminals of retinal bipolar cells has recently been shown to modulate synaptic release by hyperpolarizing the terminal (Veruki et al., 2006, Wersinger et al., 2006). The channel conductance has a chaotropic selectivity sequence: $\mathrm{SCN}^{-}>\mathrm{NO}_{3}{ }^{-}>\mathrm{I}^{-}>\mathrm{Cl}^{-}>$ $\mathrm{F}^{-}$(Wadiche et al., 1995; Eliasof and Jahr, 1996). The gating of this anion conductance has been proposed to be linked to state transitions in the glutamate transport cycle, but the small predicted unitary conductance for the channel and the transporter has not allowed a direct test of this hypothesis (Picaud et al., 1995; Larsson et al., 1996; Wadiche and Kavanaugh, 1998). The structure of a homologous archael transporter from Pyrococcus horikoshii was recently solved at $3.5 \AA$ (Yernool et al., 2004), but the structural nature of the chloride channel pore remains unclear. The transporter is a trimer of identical subunits (Fig. 1A), and each subunit appears to be able to independently transport glutamate (Awes et al., 2004; Grewer et al., 2005; Koch and Larsson, 2005). In contrast, it is less clear whether chloride permeates through each subunit or whether the chloride channel involves interacting subunits (Eskandari et al., 2000; Torres-Salazar and Fahlke, 2006), in analogy to ligand-gated channels, such as ionotropic glutamate receptors, which have a pore in the central axis of the multimer. In this work, we analyzed and modeled the isolated transport and channel conductance components and 

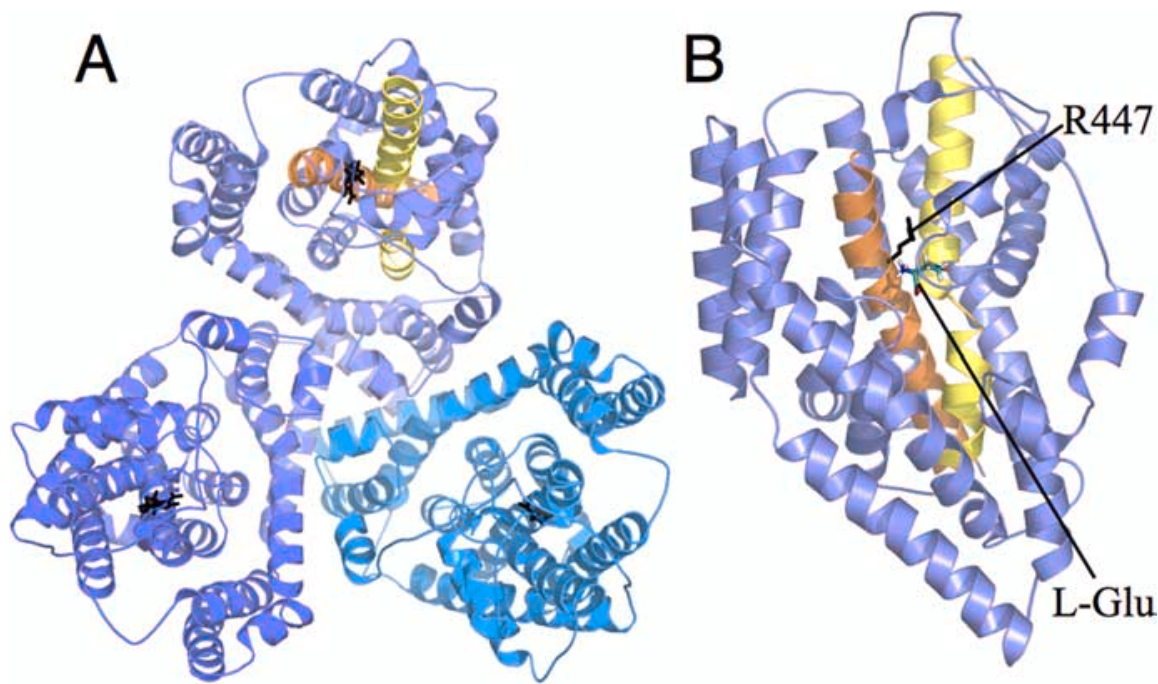

Figure 1. hEAAT3 structural homology model based on the $P$. horikoshii sodium-dependent glutamate transporter homolog structure (Yernool et al., 2004). A, Trimeric structure comprising three identical subunits. B, A single subunit showing a possible orientation of bound $\mathrm{L}-G$ lu. The R447 residue that was mutated in this study is shown in black. Homology models were constructed using SYBYL (Tripos, St. Louis, MO) and PyMOL (DeLano Scientific, Palo Alto, CA).

show that the channel gating is described by a simple model in which each subunit in a trimer has a channel that is independently gated by glutamate.

\section{Materials and Methods}

Electrophysiology and flux assays. Human EAAT3 (hEAAT3) cRNA was microinjected in stage V-VI oocytes, and transport and anion currents and radiolabel fluxes were recorded $3-5 \mathrm{~d}$ later as described previously (Wadiche et al., 1995). In coexpression experiments, equal amounts of wild-type and mutant subunit plasmid DNA were mixed before linearization and transcription. Recording solution (Cl-Ringer) contained the following (in mM): $96 \mathrm{NaCl}, 2 \mathrm{KCl}, 1 \mathrm{MgCl}_{2}, 1.8 \mathrm{CaCl}_{2}$, and $5 \mathrm{HEPES}, \mathrm{pH}$ 7.4. In chloride substitution experiments, $90 \mathrm{~mm} \mathrm{NaNO}_{3}$ was used to replace equimolar $\mathrm{NaCl}\left(\mathrm{NO}_{3}-\right.$ Ringer $)$. Microelectrodes were filled with $3 \mathrm{M} \mathrm{KCl}$ and had resistances from 1 to $3 \mathrm{MÙ}$. Two-electrode voltageclamp recordings were performed at $22^{\circ} \mathrm{C}$ with a Geneclamp 500 interfaced to an IBM-compatible personal computer using a Digidata 1320 controlled with the pCLAMP 6.0 program suite (Molecular Devices, Sunnyvale, CA). The currents were low-pass filtered at $1 \mathrm{kHz}$ and digitized at $5 \mathrm{kHz}$. Currents induced by L-Glu or L-alanine ( $\mathrm{L}-\mathrm{Ala}$ ) were isolated by subtracting currents recorded in control solution. Data were analyzed off-line, and modeling and fitting of substrate concentration dependence of the currents were performed with Kaleidagraph software (version 3.6).

Analytical modeling of the anion channel gating. Our models proceed from the assumption that one transporter subunit binds and transports glutamate independently of the others (Grewer et al., 2005; Koch and Larsson 2005; present study). Then, the unidirectional transport of substrate $S$ to release product $P$ (when $[S]_{\text {trans }}=0$ ) by each transporter subunit $T$ is described by the following:

$$
T+S \underset{k_{b}}{\stackrel{k_{f}}{\rightleftharpoons}} C \stackrel{k_{t}}{\longrightarrow} T+P
$$

where $k_{f}$ is the rate going forward, $k_{b}$ is the rate going backward, $C$ is the enzyme-substrate complex, and $k_{t}$ is the turnover number. If an independent anion channel is contained in each subunit and its open probability is increased by the binding of substrate, then, at steady state, the normalized current amplitude is given by the Michaelis-Menton relationship:

$$
\frac{I}{I_{\max }}=\frac{C}{C+T}=\frac{S}{S+K},
$$

where

$$
K=\frac{k_{b}+k_{t}}{k_{f}} .
$$

If, in contrast, an anion channel is formed by three subunits in a trimeric complex, then its open probability is increased by binding of glutamate to one, two, or three subunits. The set of transporter reaction equations defining the transporter states occupied by zero $(\mathrm{T})$, one $\left(\mathrm{C}_{1}\right)$, two $\left(\mathrm{C}_{2}\right)$, or three $\left(\mathrm{C}_{3}\right)$ molecules of glutamate is as follows:

$$
\begin{gathered}
T+S \underset{k_{b}}{\stackrel{3 k_{f}}{\rightleftharpoons}} C_{1} \stackrel{k_{t}}{\longrightarrow} T+P \\
C_{1}+S \underset{2 k_{b}}{\stackrel{2 k_{f}}{\rightleftharpoons}} C_{2} \stackrel{2 k_{t}}{\longrightarrow} C_{1}+P \\
C_{2}+S \underset{3 k_{b}}{\stackrel{k_{f}}{\rightleftharpoons}} C_{3} \stackrel{3 k_{t}}{\longrightarrow} C_{2}+P .
\end{gathered}
$$

If occupancy of only one subunit is required to open the channel, we assume that singly, doubly, and triply occupied trimers are all open; for the case in which two subunits are required, then doubly or triply occupied trimers are open; for the case in which three subunits are required, then only triply occupied trimers are open. At steady state, the normalized current amplitudes for each anion channel-gating model are as follows:

single occupancy

$$
\frac{I}{I_{\max }}=\frac{C_{1}+C_{2}+C_{3}}{C_{1}+C_{2}+C_{3}+T}=\frac{S^{3}+3 S^{2} K+3 S K^{2}}{\left(K^{3}+3 S K^{2}+3 S^{2} K+S^{3}\right)},
$$

double occupancy

$$
\frac{I}{I_{\max }}=\frac{C_{2}+C_{3}}{C_{1}+C_{2}+C_{3}+T}=\frac{S^{3}+3 S^{2} K}{\left(K^{3}+3 S K^{2}+3 S^{2} K+S^{3}\right)},
$$

triple occupancy

$$
\frac{I}{I_{\max }}=\frac{C_{3}}{C_{1}+C_{2}+C_{3}+T}=\frac{S^{3}}{\left(K^{3}+3 S K^{2}+3 S^{2} K+S^{3}\right)} .
$$

Derivations of these equations are given in the supplemental material (available at www.jneurosci.org).

\section{Results}

Each subunit in a trimer transports substrate independently Wild-type hEAAT3 subunits were coexpressed with mutant R447C subunits by injecting RNA transcribed from equal amounts of each cDNA into Xenopus laevis oocytes. The R447C mutation has been shown to induce an altered charge selectivity for neutral amino acids such as L-Ala (Bendahan et al., 2000) (Fig. 2). L-Ala $(1000 \mu \mathrm{M})$ did not activate currents in oocytes expressing wild-type hEAAT3 alone $(n=4)$, nor did L-Glu $(1000 \mu \mathrm{M})$ activate currents in oocytes expressing the R447C mutant alone $(n=3)$ (Fig. $2 A, B)$. This selectivity switch was confirmed by radiolabeled uptake assays with either $30 \mu \mathrm{M}\left[{ }^{3} \mathrm{H}\right] \mathrm{D}-\mathrm{Asp}$ or $\left[{ }^{3} \mathrm{H}\right] \mathrm{L}$-Ala (Fig. $2 \mathrm{C}$ ). Coimmunoprecipitation evidence suggests 
that wild-type and R447 mutants form heteromultimers when coexpressed in the same cells (Grewer et al., 2005). We therefore examined and compared the L-Ala and L-Glu concentration dependence of transport currents in oocytes expressing heterotrimeric wild-type hEAAT3 and R447C mutant subunits with the currents activated in homotrimeric populations of each subunit. Concentration-response data for both L-Ala and L-Glu were determined in each oocyte, and the $\mathrm{EC}_{50}$ values and Hill coefficients were measured at a series of membrane potentials between -100 and $60 \mathrm{mV}$. At $-20 \mathrm{mV}$, the reversal potential for $\mathrm{Cl}^{-}$, the current activated by glutamate predominantly reflects coupled transport (Wadiche et al. 1995) (Fig. 2A). At all membrane potentials, the L-Glu and L-Ala concentration dependence of currents in oocytes expressing heterotrimers was the same as for oocytes expressing homotrimeric wild-type and mutant transporter subunits, respectively (Fig. $2 A, B$ ) $(p>0.5)$. These data are consistent with the conclusion that a glutamate transporter resides in each subunit within the trimeric complex, because a binding site and translocation pathway formed by multiple subunits would be expected to exhibit an altered apparent affinity in the heterotrimers (Awes et al., 2004; Grewer et al., 2005; Koch and Larsson, 2005). Furthermore, because the glutamate concentration dependence of transport exhibited a Hill coefficient $\leq 1$, each subunit of the trimer appears to function independently.

\section{Analytical modeling of anion channel gating}

We considered two general classes of models for anion channel gating in a trimeric molecule: (1) a channel within each subunit that is activated by glutamate binding or (2) a single channel that is gated by glutamate binding to one, two, or three subunits. Using the intrinsic transport kinetic parameter $K$ estimated from measurement of the $\mathrm{EC}_{50}$ of glutamate transport in the absence of anion flux $(44 \mu \mathrm{M})$ (see Fig. $4 A$ ), we derived the predicted concentration-dependent conductance of the anion channel for each of the models. This estimated value was in good agreement with previous microscopic rate measurements and modeling, with a glutamate-binding rate of $6.8 \times 10^{6} \mathrm{M}^{-1} \mathrm{~s}^{-1}$, an unbinding rate of $300 \mathrm{~s}^{-1}$, and a transport rate of $14.6 \mathrm{~s}^{-1}$ (Larsson et al., 2004; Wadiche et al., 1995),

$$
K=\frac{k_{b}+k_{t}}{k_{f}}=46 \mu \mathrm{M}
$$

(also see Materials and Methods and supplemental material, available at www.jneurosci.org). This value was used to derive the predicted glutamate concentration dependence of the channel conductance for the one-channel/one-subunit model as well as for the three models in which a central channel is gated by glutamate binding to one, two, or three subunits. For a given transport constant $K$, which reflects glutamate binding, unbinding, and transport rates, each channel-gating model predicts a unique concentration dependence of anion channel activation that can be experimentally tested to identify the gating mechanism of the anion channel (Fig. 3).

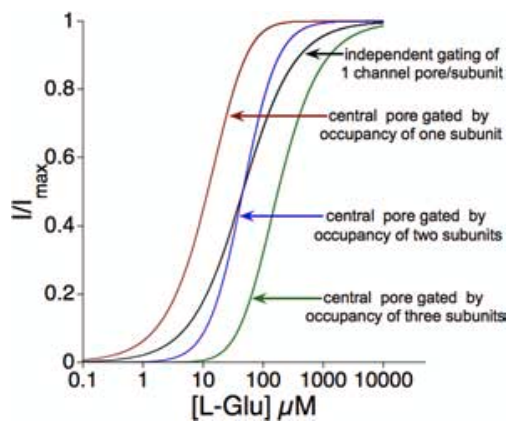

Figure 3. Analytical modeling of anion channel gating. Shown are models depicting anion channel schemes for the following: (1) independent gating in each subunit of the trimer (black line; $\left.\mathrm{EC}_{50}=45 \mu \mathrm{m} ; n_{\text {Hill }}=1\right)$, (2) central pore gated by noncooperative binding to one or more subunits (red line; $\mathrm{EC}_{50}=11.90 ; n_{\text {Hill }}=1.48$ ), (3) central pore gated by occupancy of two or more subunits (blue line; $\mathrm{EC}_{50}=46.33 ; n_{\text {Hill }}=1.57$ ), and (4) central pore gated by occupancy of three subunits (green line; $\mathrm{EC}_{50}=180.50 ; n_{\text {Hill }}=1.18$ ).

\section{The isolated anion current is best fit by the model of one channel within each subunit}

The four models represented in Figure 3 predict distinct differences in anion channel gating as a function of glutamate concentration. Because the presence of the permeant anion $\mathrm{NO}_{3}$ does not affect the rate of coupled transport (Wadiche and Kavanaugh, 1998), the anion current was isolated by subtracting the coupled transport current (recorded in Cl-Ringer at an $E_{\mathrm{Cl}}$ of $-20 \mathrm{mV}$ ) from the current recorded at the same potential after switching the external solution to $\mathrm{NO}_{3}$-Ringer. Figure $4 \mathrm{~A}$ shows the $\mathrm{L}-\mathrm{Glu}$ dependence of both the anion current and the coupled transport current at $-20 \mathrm{mV}$. The concentration dependence of anion channel activation was compared with each model and quantified by $\chi^{2}$ measurement (Fig. $4 B$ ). The data are best fit by the model in which one anion channel within each subunit is gated independently $\left(+^{2}=8.08\right)$. The next best fit was for the model in which the anion conductance is gated by binding of glutamate to at least two subunits $\left(+^{2}=202\right)$. There was no 

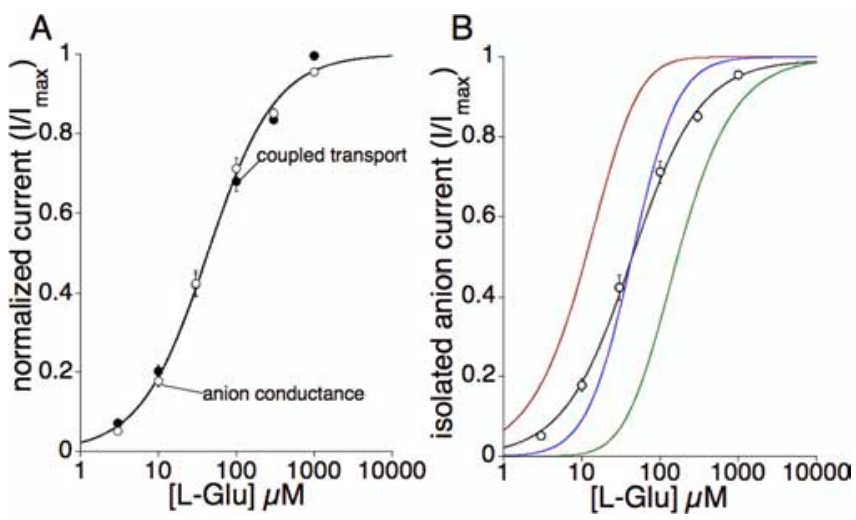

Figure 4. Modeling of channel activation data. $\boldsymbol{A}$, Concentration dependencies of isolated coupled transport current $\left(\mathrm{EC}_{50}=43.7 \mu \mathrm{m} ; n=8 ; \mathrm{Cl}^{-}\right.$-Ringer, $\left.-20 \mathrm{mV}\right)$ and the uncoupled anion current $\left(\mathrm{O} ; \mathrm{EC}_{50}=42.8 ; n=8 ; \mathrm{NO}_{3}-\right.$ Ringer, $\left.-20 \mathrm{mV}\right)$. The coupled and anion currents were fitted to $\left(I / I_{\max }=[\mathrm{L}-\mathrm{Glu}] /[\mathrm{L}-\mathrm{Glu}]+\mathrm{EC}_{50}\right) . \boldsymbol{B}$, The model predictions and the experimental anion conductance data are shown for an anion channel within each subunit (black line; $\chi^{2}=8.08$ ), one central anion pore gated by noncooperative binding to one or more subunits (red line; $\chi^{2}=1369$ ), one central anion pore gated by noncooperative binding to at least two subunits (blue line; $\chi^{2}=202$ ), or one central anion pore gated by noncooperative binding of all three subunits (green line; $\chi^{2}=867$ ). The measured coupled transport constant used for the fitting was $44 \mu \mathrm{m}$. Error bars indicate SEM.

increase in apparent glutamate cooperativity at potentials up to $+60 \mathrm{mV}(n=1.08 \pm 0.03 ; n=9)$.

\section{Discussion}

Data from several laboratories obtained by coexpression of wildtype and mutant subunits is consistent with the conclusion that glutamate binds and translocates through each of the single subunits in a trimeric complex (Awes et al., 2004; Grewer et al., 2005; Koch and Larsson, 2005). In agreement with Grewer et al. (2005), we found that when wild-type subunits are coexpressed with mutant subunits, two independent populations of transporters are observed. Assuming that a fraction of the trimers are heteromers (Grewer et al., 2005), this result suggests that each subunit transports substrate independently. Important caveats arise when making quantitative inferences using this experimental approach because of untested assumptions of random translation and interaction of wild-type and mutant subunits expressed at the plasma membrane as well as uncertainties about the microscopic conductance properties of the various homotrimers and heterotrimers. To circumvent these caveats, we applied MichaelisMenten analysis to homotrimeric wild-type transporters and derived equations that predict the distribution of singly, doubly, and triply occupied trimeric complexes as a function of glutamate concentration. These derivations depend only on the assumption that each subunit transports glutamate independently (Awes et al., 2004; Grewer et al., 2005; Koch and Larsson, 2005; Leary et al., 2006).

We find that the glutamate concentration dependence of the fractional anion conductance is the same as the fraction of total subunits occupied by glutamate, consistent with the presence of a channel in each subunit that is gated by glutamate binding. This is in contrast to the predictions for gating of a central channel controlled by occupancy of one, two, or three subunits. A consequence of the one-subunit/one-channel gating scheme is that the concentration dependencies of transport and anion conductance are expected to be identical, which is indeed observed (Fig. 4A). This result conflicts with that of Torres-Salazar et al. (2006), who found evidence of subunit cooperativity in a recent study of chan- nel gating of the related EAAT4 glutamate transporter. Aside from the difference in transporter subtype, it is presently unclear why these results differ. It is possible that in the recording conditions of Torres-Salazar et al. (2006), which used intracellular $\mathrm{Na}^{+}$ and involved activation of transient anion currents by voltage jumps, additional conductances could be reflected in the presteady-state currents, which are not monitored in the steadystate conditions studied here.

The detailed molecular mechanisms controlling the chloride channel functions of glutamate transporters are still unclear. A cluster of residues in the second transmembrane domain (TMD2) that affects anion permeation has been identified by Ryan et al. (2004). These residues are in a helix that is adjacent to the likely glutamate- binding site (Fig. $1 B$ ). TMD8, where R447 lies, and TMD7, where other residues implicated in alkali cation coupling lie (Kavanaugh et al., 1997), are also close to the likely glutamate-binding site. The identity of the coupled alkali cation also strongly influences the anion conductance (Borre and Kanner, 2001), suggesting that these helices could potentially be involved in forming a permeation path that could be shared by $\mathrm{L}-\mathrm{Glu}$, the coupled cations, and $\mathrm{Cl}^{-}$ions. Examples of stochastic gating and high substrate flux rates in other transporters support the idea that a transporter pore may be subject to channel-like gating modes that are incongruous with conventional alternating access gating (DeFelice and Goswami, 2006).

An additional unresolved question concerns the reason for the trimeric nature of the transporter given that the subunits function independently. It is unknown whether dissociated monomeric subunits are functional, but it is possible that a multimeric structure has evolved to play a functional role (e.g., a large aqueous bowl projecting partially through the plane of the membrane might facilitate transport of charged substrates across the membrane dielectric). Analysis of the quaternary structure and channel properties of other members of this transporter superfamily may shed additional light on these issues.

\section{References}

Awes AN, Hubbard J, Kanner BI, Kavanaugh MP (2004) Structure of the selectivity filter in the glutamate/neutral amino acid transporter family. Soc Neurosci Abstr 30:168.13.

Bendahan A, Armon A, Madani N, Kavanaugh MP, Kanner BI (2000) Arginine 447 plays a pivotal role in substrate interactions in a neuronal glutamate transporter. J Biol Chem 275:37436-37442.

Billups B, Rossi D, Attwell D (1996) Anion conductance behavior of the glutamate uptake carrier in salamander retinal glial cells. J Neurosci 16:6722-6731.

Borre L, Kanner BI (2001) Coupled, but not uncoupled, fluxes in a neuronal glutamate transporter can be activated by lithium ions. J Biol Chem 276:40396-40401.

Danbolt NC (2001) Glutamate uptake. Prog Neurobiol 65:1-105.

DeFelice LJ, Goswami T (2007) Transporters as channels. Annu Rev Physiol 69:87-112.

Eliasof S, Jahr CE (1996) Retinal glial cell glutamate transporter is coupled to an anionic conductance. Proc Natl Acad Sci USA 93:4153-4158.

Eskandari S, Kreman M, Kavanaugh MP, Wright EM, Zampighi GA (2000) Pentameric assembly of a neuronal glutamate transporter. Proc Natl Acad Sci USA 97:8641-8646.

Fairman WA, Vandenberg RJ, Arriza JL, Kavanaugh MP, Amara SG (1995) An excitatory amino-acid transporter with properties of a ligand-gated chloride channel. Nature 375:599-603.

Grewer C, Balani P, Weidenfeller C, Bartusel T, Tao Z, Rauen T (2005) Individual subunits of the glutamate transporter EAAC1 homotrimer function independently of each other. Biochemistry 44:11913-11923.

Kavanaugh MP, Bendahan A, Zerangue N, Zhang Y, Kanner BI (1997) Mutation of an amino acid residue influencing potassium coupling in the glutamate transporter GLT-1 induces obligate exchange. J Biol Chem 272:1703-1708. 
Koch HP, Larsson HP (2005) Small-scale molecular motions accomplish glutamate uptake in human glutamate transporters. J Neurosci 25:1730-1736.

Larsson HP, Picaud SA, Werblin FS, Lecar H (1996) Noise analysis of the glutamate-activated current in photoreceptors. Biophys J 70:733-742.

Larsson HP, Tzingounis AV, Koch HP, Kavanaugh MP (2004) Fluorometric measurements of conformational changes in glutamate transporters. Proc Natl Acad Sci USA 101:3951-3956.

Levy LM, Warr O, Attwell D (1998) Stoichiometry of the glial glutamate transporterGLT-1 expressed inducibly in a Chinese hamster ovary cell line selected for low endogenous $\mathrm{Na}+$-dependent glutamate uptake. J Neurosci 18:9620-9628.

Picaud SA, Larsson HP, Grant GB, Lecar H, Werblin FS (1995) Glutamategated chloride channel with glutamate-transporter-like properties in cone photoreceptors of the tiger salamander. J Neurophysiol 74:1760-1771.

Ryan RM, Mitrovic AD, Vandenberg RJ (2004) The chloride permeation pathway of a glutamate transporter and its proximity to the glutamate translocation pathway. J Biol Chem 279:20742-20751.
Torres-Salazar D, Fahlke C (2006) Intersubunit interactions in EAAT4 glutamate transporters. J Neurosci 26:7513-7522.

Veruki ML, Mørkve SH, Hartveit E (2006) Activation of a presynaptic glutamate transporter regulates synaptic transmission through electrical signaling. Nat Neurosci 9:1388-1396.

Wadiche JI, Kavanaugh MP (1998) Macroscopic and microscopic properties of a cloned glutamate transporter/chloride channel. J Neurosci 18:7650-7661.

Wadiche JI, Amara SG, Kavanaugh MP (1995) Ion fluxes associated with excitatory amino acid transport. Neuron 15:721-728.

Wersinger E, Schwab Y, Sahe JA, Rendon A, Pow DV, Picaud S, Roux MJ (2006) The glutamate transporter EAAT5 works as a presynaptic receptor in mouse rod bipolar cells. J Physiol (Lond) 577:221-234.

Yernool D, Boudker O, Jin Y, Gouaux E (2004) Structure of a glutamate transporter homologue from Pyrococcus horikoshii. Nature 431: 811-818.

Zerangue N, Kavanaugh MP (1996) Flux coupling in a neuronal glutamate transporter. Nature 383:634-637. 